\title{
Aedes aegypti e fauna associada em área rural de Manaus, na Amazônia brasileira
}

\author{
Aedes aegypti and associated fauna in the rural zone of Manaus, \\ in the Brazilian Amazon
}

\author{
Maria Graças Vale Barbosa ${ }^{1,2,3}$, Nelson Ferreira Fé1, Rossicleide Dias Barbosa de Jesus ${ }^{1}$, \\ Iria Cabral Rodriguez ${ }^{1}$, Wuelton Marcelo Monteiro, ${ }^{1,2}$, Maria Paula Gomes Mourão ${ }^{1,2,3}$ \\ e Jorge Augusto de Oliveira Guerra ${ }^{1,2}$
}

\begin{abstract}
RESUMO
Após detectar larvas de Aedes aegypti em área rural de Manaus, realizou-se durante dois anos consecutivos um trabalho de notificação da ocorrência dessa espécie naquela área, através da vigilância entomológica, ferramenta que representa um importante papel entre as medidas preventivas contra doenças de transmissão por insetos em particular as arboviroses.
\end{abstract}

Palavras-chaves: Aedes aegypti. Aedes albopictus. Arboviroses.

\begin{abstract}
After detecting Aedes aegypti larvae in the rural zone of Manaus, entomological surveillance was carried out over two consecutive years in order to notify occurrences of this species in that area. The tool of entomological surveillance has an important role among the preventive measures against diseases transmitted by insects, particularly arbovirosis.
\end{abstract}

Key-words: Aedes aegypti. Aedes albopictus. Arbovirosis.

Considerada a arbovirose mais importante do mundo, a reemergência da dengue está diretamente relacionada à reinfestação das áreas urbanas pelo Aedes aegypti $i^{10}$, espécie que por ser disseminada principalmente de forma passiva, tem muitas vezes, a sua distribuição geográfica descontínua ${ }^{6}$, relacionada a condições climáticas e disponibilidade de ecótopos adequados para 0 estabelecimento de criadouros ${ }^{2}$. A ocorrência deAedes aegypti nos ambientes urbanos tem sido relatada na literatura ${ }^{2}$. Entretanto, em áreas rurais, especialmente na Região Amazônica, as informações são escassas. 0 primeiro relato de sua ocorrência na área urbana de Manaus, Estado do Amazonas, deu-se em 19965, e em 2002, em área rural $^{1}$, o que motivou esse estudo para registrar o estabelecimento de Aedes aegypti, e a fauna de culicídeos associada, em uma área rural do município de Manaus.

0 trabalho foi realizado em parceria com a Secretaria de Estado de Saúde do Amazonas, entre junho de 2002 e julho de 2004, na Comunidade Nossa Senhora de Fátima, área margeada

1.Gerências de Entomologia, Fundação de Medicina Tropical do Amazonas, Manaus, AM, 2. Escola Superior de Ciências da Saúde, Universidade do Estado do Amazonas, Manaus, AM. 3. Centro Universitário Nilton Lins, Manaus, AM.

Endereço para correspondência: Dra. Maria das Graças Vale Barbosa. Gerência de Entomologia/FMTAM. Av. Pedro Teixeira 25, Dom Pedro, 69040-000 Manaus, AM.

Fax: 5592 2127-3525.

e-mail: gbarbosa@fmt.am.gov.br

Recebido em 25/09/2008

Aceito em 05/03/3009 pela floresta, localizada a oeste desta capital, a $3^{\circ} 01$ ' 17 'S; $60^{\circ} 09^{\prime}$ 51'W, cujo acesso se dá preferencialmente por via fluvial. Dentro da comunidade, na área externa e interna de sete residências, foram instaladas 14 ovitrampas e 14 larvitrampas, distribuídas em pontos estratégicos, inspecionadas a cada sete dias.

Foram coletadas 8.931 formas imaturas de culicídeos, sendo 3.207 (35,9\%) nas larvitrampas e 5.724 (64,1\%) nas ovitrampas e (Tabela 1). Do total, 5.037 (56,4\%) exemplares foram de Aedes aegypti, $1.304(25,9 \%)$ coletados nas larvitrampas (528 ovos aderidos à superfície interna do pneu, 724 larvas, e 52 pupas) e 3.733 (74,1\%) nas ovitrampas (3.017 ovos, 711 larvas e 5 pupas). De maio a dezembro de 2003 e no último trimestre do estudo, não se coletou Aedes aegypti (Figura 1). Entre janeiro e julho de 2004, houve registro de 111 exemplares de Aedes albopictus, $110(99,1 \%)$ nas larvitrampas (100 larvas e 10 pupas) e uma (0,9\%) larva em ovitrampa.

Durante o trabalho, foram coletadas e identificadas 659 larvas de Toxorhynchites haemorroidales, 391 deLimatus durhami, 323 de Trichoprosopon digitatum, 210 de Culex quinquefasciatus, 25 de Haemagogus baresi, 14 de Wyeomyia oculta, 3 de Culex nigripalpus, 1 de Haemagogus leucocelaenus, 1 de Aedes fluviatilis e 1 de Psorophora amazonica. Entretanto, 2.153 larvas de Culex sp não foram identificadas a nível específico, porque se encontravam no primeiro estádio.

0 registro de Aedes aegypti na área de estudo aconteceu em março de 2002, em recipientes artificiais e naturais, entre os 
naturais, ouriço de castanha-do-pará (Bertholletia excelsa) e casca de cupuaçu (Theobroma grandiflorum), durante atividades de busca ativa de casos de malária ${ }^{1}$. Na ocasião, foram atendidas pessoas com síndrome febril exantemática aguda com pesquisa de plasmódio negativa, sendo os casos suspeitos encaminhados para atendimento na Fundação de Medicina Tropical do Amazonas. Entre os casos investigados por sorologia (MAC-ELISA), foram identificados nove casos de infecção aguda pelo vírus dengue, todos sem gravidade e com história prévia de deslocamento para
Manaus. Esse fato, aliado ao encontro de larvas de Aedes aegypti, motivou a realização deste estudo. 0 encontro de ovos dessa espécie e de larvas deAedes albopictus em larvitrampas confirma suas capacidades de adaptação e associação em diferentes ambientes e criadouros ${ }^{247}$, evidenciando o papel da transposição antrópica dessas espécies da área urbana para a área rural, com a possibilidade de estabelecimento nas comunidades rurais da Amazônia, onde existe uma rica diversidade de espécies de vírus associados a doenças humanas ${ }^{237}$.

\section{TABELA 1}

Aedes aegypti e fauna de culicídeos associada, coletados entre junho de 2002 e julho de 2004 na zona rural do município de Manaus, Amazonas.

\begin{tabular}{|c|c|c|c|c|c|c|c|c|c|c|}
\hline \multirow[b]{2}{*}{ Espécies } & \multicolumn{4}{|c|}{ Larvitrampa } & \multicolumn{4}{|c|}{ Ovitrampa } & \multicolumn{2}{|c|}{ Total geral } \\
\hline & ovo & larva & pupa & total & ovo & larva & pupa & total & $\mathrm{n}^{\underline{0}}$ & $\%$ \\
\hline \multicolumn{11}{|l|}{2002} \\
\hline Aedes aegypti & 388 & 278 & 21 & 687 & 2.012 & 376 & 5 & 2.393 & 3.080 & 34,5 \\
\hline Culex spp & 0 & 61 & 0 & 61 & 50 & 14 & 0 & 64 & 125 & 1,4 \\
\hline Culex quinquefasciatus & 7 & 2 & 0 & 9 & 100 & 101 & 0 & 201 & 210 & 2,4 \\
\hline Haemagogus bares $i$ & 0 & 6 & 0 & 6 & 0 & 0 & 0 & 0 & 6 & 0,1 \\
\hline Limatus durhami & 0 & 13 & 0 & 13 & 0 & 0 & 0 & 0 & 13 & 0,2 \\
\hline Toxorhynchites haemorroidales & 0 & 259 & 0 & 259 & 4 & 32 & 0 & 36 & 295 & 3,3 \\
\hline Trichoprosopon digitatum & 0 & 10 & 0 & 10 & 0 & 1 & 0 & 1 & 11 & 0,1 \\
\hline Total 2002 & 395 & 629 & 21 & 1.045 & 2.166 & 524 & 5 & 2.695 & 3.740 & 41,9 \\
\hline \multicolumn{11}{|l|}{2003} \\
\hline Aedes aegypti & 140 & 427 & 31 & 598 & 923 & 328 & 0 & 1.251 & 1.849 & 20,7 \\
\hline Culex spp & 0 & 374 & 0 & 374 & 379 & 819 & 0 & 1.198 & 1.572 & 17,6 \\
\hline Culex nigripalpus & 0 & 0 & 0 & 0 & 3 & 0 & 0 & 3 & 3 & 0,0 \\
\hline Haemagogus baresi & 0 & 3 & 0 & 3 & 3 & 0 & 0 & 3 & 6 & 0,1 \\
\hline Limatus durhami & 0 & 73 & 6 & 79 & 0 & 0 & 0 & 0 & 79 & 0,9 \\
\hline Psorophora amazonica & 0 & 1 & 0 & 1 & 0 & 0 & 0 & 0 & 1 & 0,0 \\
\hline Toxorhynchites haemorroidales & 0 & 203 & 3 & 206 & 3 & 18 & 0 & 21 & 227 & 2,5 \\
\hline Trichoprosopon digitatum & 0 & 6 & 0 & 6 & 0 & 0 & 0 & 0 & 6 & 0,1 \\
\hline Wyeomyia oculta & 0 & 8 & 0 & 8 & 0 & 1 & 0 & 1 & 9 & 0,1 \\
\hline Total 2003 & 140 & 1.095 & 40 & 1.275 & 1.311 & 1.166 & 0 & 2.477 & 3.752 & 42,0 \\
\hline \multicolumn{11}{|l|}{2004} \\
\hline Aedes aegypti & 0 & 19 & 0 & 19 & 82 & 7 & 0 & 89 & 108 & 1,2 \\
\hline Aedes albopictus & 0 & 100 & 10 & 110 & 0 & 1 & 0 & 1 & 111 & 1,2 \\
\hline Aedes argyrothorax & 0 & 1 & 0 & 1 & 0 & 0 & 0 & 0 & 1 & 0,0 \\
\hline Aedes fluviatilis & 0 & 1 & 0 & 1 & 0 & 0 & 0 & 0 & 1 & 0,0 \\
\hline Culex spp & 0 & 72 & 0 & 72 & 0 & 384 & 0 & 384 & 456 & 5,1 \\
\hline Haemagogus baresi & 0 & 13 & 1 & 14 & 0 & 0 & 0 & 0 & 14 & 0,2 \\
\hline Haemagogus leucocelaenus & 0 & 1 & 0 & 1 & 0 & 0 & 0 & 0 & 1 & 0,0 \\
\hline Limatus durhami & 0 & 221 & 18 & 239 & 0 & 60 & 0 & 60 & 299 & 3,4 \\
\hline Toxorhynchites haemorroidales & 0 & 118 & 1 & 119 & 0 & 18 & 0 & 18 & 137 & 1,5 \\
\hline Trichoprosopon digitatum & 0 & 306 & 0 & 306 & 0 & 0 & 0 & 0 & 306 & 3,4 \\
\hline Wyeomyia oculta & 0 & 5 & 0 & 5 & 0 & 0 & 0 & 0 & 5 & 0,1 \\
\hline Total 2004 & 0 & 857 & 30 & 887 & 82 & 470 & 0 & 552 & 1.439 & 16,1 \\
\hline Total geral & 535 & 2.581 & 91 & 3.207 & 3.559 & 2.160 & 5 & 5.724 & 8.931 & 100 \\
\hline$\%$ & 6,0 & 28,9 & 1,0 & 35,9 & 39,8 & 24,2 & 0,1 & 64,1 & 100 & \\
\hline \multirow[t]{3}{*}{ Aedes aegypti } & 388 & 278 & 21 & 687 & 2012 & 376 & 5 & 2.393 & 3.080 & 61,2 \\
\hline & 140 & 427 & 31 & 598 & 923 & 328 & 0 & 1.251 & 1.849 & 36,7 \\
\hline & 0 & 19 & 0 & 19 & 82 & 7 & 0 & 89 & 108 & 2,14 \\
\hline Total & 528 & 724 & 52 & 1.304 & 3.017 & 711 & 5 & 3.733 & $5.037 / 8.931$ & \\
\hline$\%$ & 10,5 & 14,4 & 1,03 & 25,9 & 59,9 & 14,1 & 0,1 & 74,1 & 56,4 & \\
\hline
\end{tabular}




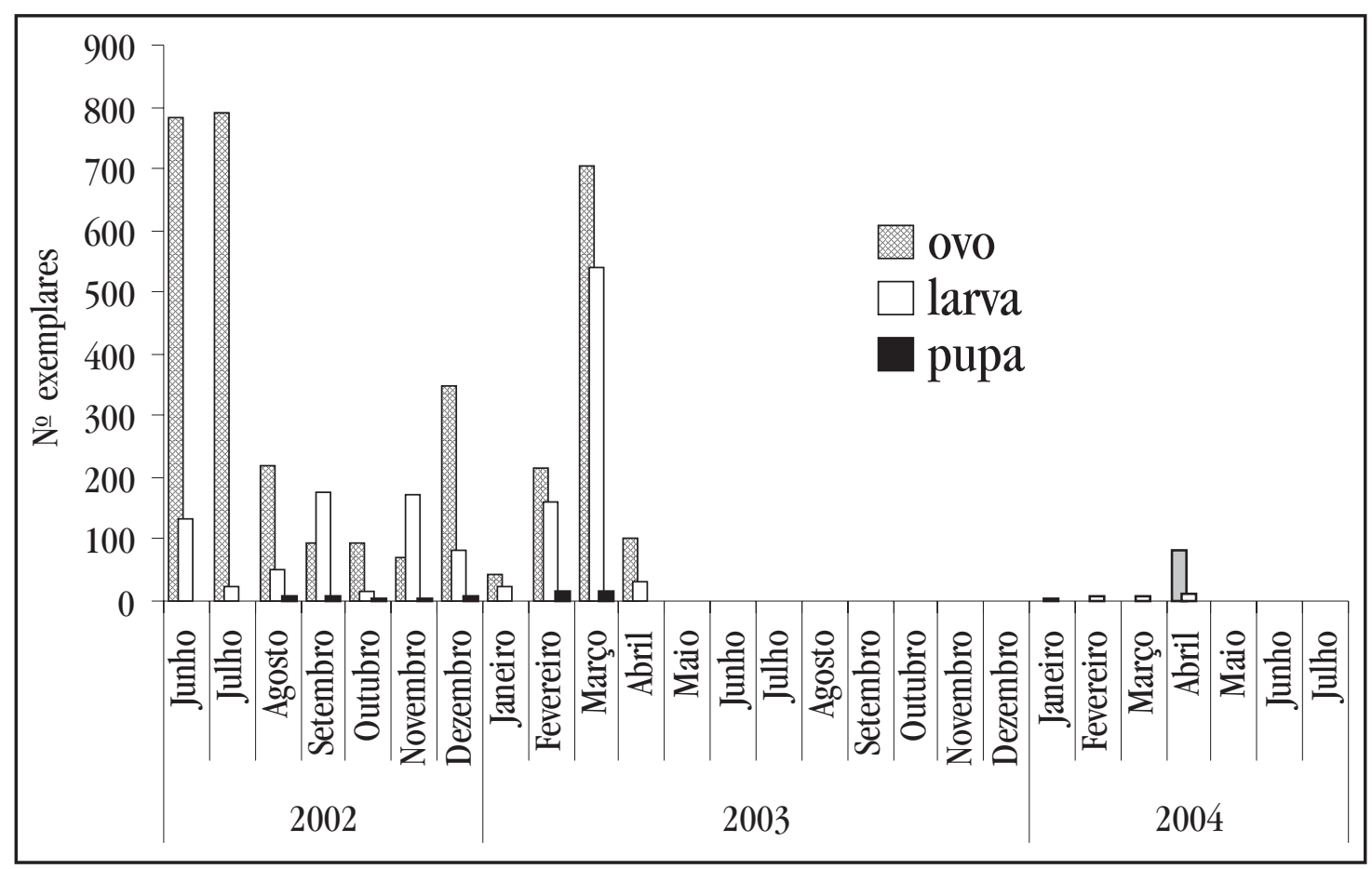

FIGURA 1

Variação mensal da abundância de imaturos de Aedes aegypti coletados entre junho de 2002 e julho de 2004 na zona rural do município de Manaus, Amazonas.

A capacidade de procriação de Aedes aegypti e Aedes albopictus em distintos gradientes ecológicos e a ausência de competidores autóctones, favorece sua adaptação, quer por condições ecológicas adequadas ${ }^{8}$, ou sua flexibilidade genética 9 . Essa adaptabilidade representa impacto à saúde pública, face ao risco potencial dessas espécies virem a exercer papel vetorial para diversas arboviroses. Nesse sentido, faz-se necessária a adoção de medidas preventivas como mecanismo que permitam acompanhar o estado de saúde dessa população em relação às arboviroses, principalmente porque houve registro da ocorrência de espécies de culicideos estritamente silvestres, de grande importância epidemiológica, por serem considerados vetores potenciais na transmissão de arboviroses, filarioses e outros patógenos e fatores de incômodo para o homem. Como exemplo, Haemagogus leucocelaenus incriminado como vetor primário da febre amarela silvestre no sudeste do $\mathrm{Brasil}^{2}$, Aedes fluviatilis mosquito comum em locais silvestres, semi-silvestres suburbanos e urbanos, que já demonstrou capacidade de infectarse experimentalmente com vírus da febre amarela e Dirofilaria immitis $^{2}$, encontrado em recipiente artificiais em associação com Aedes aegypti ou Culex quinquefasciatus ${ }^{2}$. Esta última, é uma espécie beneficiada pelas alterações antrópicas no ambiente peridomiciliar ${ }^{27}$ considerada 0 mais eficiente vetor da filariose bancroftiana ao homem, incriminada na transmissão da encefalite de Saint Loius e com registro de infecção natural pelo arbovirus Oropouche. 0 registro de Limatus durhami ${ }^{2}$ e Trichoprosopon digitatum $^{11}$ infere a possibilidade de comportamento oportunista, pela capacidade de adaptação a diferentes condições que lhe são impostas. Além disto, registrou-se a ocorrência de Culex nigripalpus, espécie incriminada na transmissão da encefalite de Saint Louis ${ }^{7}$ e Psorophora amazonica, cujo gênero contribui para a disseminação de arbovírus em condições naturais, a exemplo dos vírus Ilhéus, Mayaro, encefalite equiina Oeste, Rocio, encefalite equiina Venezuelana e outros ${ }^{3}$. Ressalta-se, ainda, a presença de fauna associada predadora (larvas de Toxorhynchites haemorroidale $^{27}$ ) que provavelmente exerceram pressão na regulação da população das outras espécies, influenciando o número de indivíduos.

0 estabelecimento de Aedes aegypti e Aedes albopictus na comunidade pode ser uma tentativa de dispersão populacional a partir de focos urbanos da Cidade de Manaus e guarde relação com a mobilidade populacional, pois muitos moradores se deslocam diariamente, de forma oscilante, para a zona urbana de Manaus, buscando trabalho, atendimento à saúde e compra de mercadorias de subsistência. Embora esta observação não precise o local de infecção dos casos registrados entre os moradores, a incursão de portadores do vírus na comunidade, mesmo que a infecção não seja autóctone, e a presença de vetores, evidencia a vulnerabilidade dessa população. Durante o período de estudo, nenhum caso novo de dengue foi identificado na área, provavelmente pela eliminação do foco inicial e pela redução significativa de imaturos capturados pelas ovitrampas, entretanto novos casos de dengue voltaram a ser identificados na área a partir de 2006.

\section{AGRADECIMENTOS}

Os autores agradecem o suporte recebido da Secretaria Estadual de Saúde do Amazonas, técnicos da Gerência de Entomologia da Fundação de Medicina Tropical do Amazonas e demais colaboradores. 


\section{REFERÊNCIAS}

1. Barbosa MGV, Fé NF, Alencar ICA, Fernandez IC, Gimaque JBL, Pastor OF, Fé FAA, Alecrim WD, Guerra MVF. Ocorrência de Aedes aegypti em áreas com características rurais dentro do município de Manaus, Amazonas, Brasil (Dados preliminares). Revista da Sociedade Brasileira de Medicina Tropical 36: 413, 2003 .

2. Consoli RAGB, Lourenço-de-Oliveira R. Principais Mosquitos de Importância Sanitária no Brasil. Editora da Fundação Oswaldo Cruz, Rio de Janeiro, 1994.

3. Dégallier N, Hervé JP, Travassos da Rosa APA, Travassos da Rosa ES, Vasconcelos PFC, Monteiro HAO, Sá Filho G, Travassos da Rosa JFS. Entomological studies on Dengue fever vectors in Brazil: the epidemics of Boa Vista, Roraima, 1982, Niterói, Rio de Janeiro, 1986, and Ceará State, 1986, 1994. In: Travassos APA, Vasconcelos PFC, Travassos da Rosa JFST (eds) An overview of arbovirology in Brazil and neighboring countries. Instituto Evandro Chagas, Belém, p.261-271, 1998.

4. Edman JD, Scott TW, Costero A, Morrison AC, Harrington LC, Clark GG. Aedes aegypti (Diptera: Culicidae) movement influenced by availability of ovipostition sites. Journal of Medical Entomology 35: 578-583, 1998.
5. Fé NF, Barbosa MGV, Alecrim WD, Guerra MVF. Registro da ocorrência de Aedes albopictus em zona urbana de Manaus, Amazonas, Brazil. Revista de Saúde Pública 37: 1-4, 2003.

6. Figueiredo RMP, Thatcher BD, Lima ML, Almeida TC, Alecrim WD, Guerra MVF. Doenças exantemáticas e primeira epidemia de dengue ocorrida em Manaus, Amazonas, no período de 1998-1999. Revista da Sociedade Brasileira de Medicina Tropical 37: 476-479, 2004.

7. Forattini OP. Culicidologia Médica. Editora da Universidade de São Paulo, São Paulo, 2003.

8. Gomes AC, Forattini OP, Kakitani I, Marques GRAM, Marques CCA, Marucci D, Brito M. Microhabitats de Aedes albopictus (Skuse) na região do Vale do Paraíba, Estado de São Paulo, Brasil. Revista de Saúde Pública 26: 108-118, 1992.

9. Rai KS. Genetics of Aedes albopictus. Journal of the American Mosquito Control Association 2: 429-436, 1986.

10. Tauil PL. Aspectos críticos do controle do dengue no Brasil. Cadernos de Saúde Pública 18: 867-871, 2002.

11. Zavortink TJ, Roberts DR, Hoch AL. Trichoprosopon digitatum - Morphology, Biology and Potential Medical Importance. Mosquito Systematics 15: 141-149, 1983. 